\title{
ARTIGOS
}

Recebido em 23.04.2013. Aprovado em 27.09.2013

Avaliado pelo sistema double blind review. Editora Científica: Fernanda Finotti Cordeiro Perobelli

http://dx.doi.org/10.1590/So034-759020140406

\section{OTIMIZAÇÃO DE PORTFÓLIOS: ANÁLISE DE EFICIÊNCIA}

\author{
Portfolio optimization: efficiency analysis \\ Optimización de portafolios: análisis de eficiencia
}

\section{PAULO ROTELA JUNIOR \\ paulo.rotela@gmail.com Mestre em Engenharia de Produção pelo Instituto de Engenharia de Produção e Gestão, Universidade Federal de Itajubá, Itajubá, MG - Brasil}

\section{EDSON DE OLIVEIRA PAMPLONA}

pamplona@unifei.edu.br

Professor do Instituto de Engenharia de Produção e Gestão, Universidade Federal de Itajubá, Itajubá, MG Brasil

\section{FERNANDO LUIZ RIÊRA SALOMON}

fer.salomon@hotmail.com

Mestrando em Engenharia de Produção pelo Instituto de Engenharia de Produção e Gestão, Universidade Federal de Itajubá, Itajubá, MG - Brasil

\section{RESUMO}

Este artigo tem como objetivo analisar o comportamento de uma carteira de ativos selecionados por meio da Análise Envoltória de Dados (DEA), otimizada pela abordagem de Sharpe, e compará-la a carteiras de ativos obtidas somente com a DEA ou abordagem de Sharpe. Para isso, utilizou-se o modelo da DEA para avaliar a eficiência de ações da Bolsa de Valores de São Paulo (Bovespa), empregando retorno, variância e outros indicadores, como variáveis de entrada e saída. Utilizou-se, ainda, a abordagem de Sharpe para otimizar a composição da carteira. Na comparação das carteiras, observa-se que a resultante da combinação de ambos os modelos apresentou melhor desempenho do que as carteiras otimizadas por apenas um dos modelos.

PALAVRAS-CHAVE | Seleção de portfólios, otimização multiobjetivo, Análise Envoltória de Dados, indicadores, Índice Sharpe.

\section{ABSTRACT}

This article aims to analyze the behavior of a portfolio of assets selected by Data Envelopment Analysis (DEA), optimized by the Sharpe approach, and compare it to portfolios of assets obtained only by DEA or the Sharpe approach. To do that, we used the DEA model to assess the efficiency of shares of the São Paulo Stock Exchange (Bovespa), employing return, variance and other indicators such as input and output variables. Also, we used the Sharpe approach to optimize the portfolio composition. In the comparison of portfolios, we noted that the resulting combination of both models performed better than the portfolios optimized by only one of the models.

KEYBOARDS / Portfolio selection, multi-objective optimization, Data Envelopment Analysis, Indicators, Sharpe Index.

\section{RESUMEN}

Este artículo tiene por objetivo analizar el comportamiento de una cartera de activos seleccionados por medio del Análisis del Grupo de Datos (DEA), optimizada por el abordaje de Sharpe, y compararla a carteras de activos obtenidas solamente con el DEA o por un abordaje de Sharpe. Para esto, se utilizó el modelo de DEA para evaluar la eficiencia de acciones de la Bolsa de Valores de São Paulo (Bovespa), empleando retorno, variancia y otros indicadores, como variables de entrada y salida. Se utilizó, también, el abordaje de Sharpe para optimizar la composición de la cartera. En la comparación de las carteras, se observa que el resultado de la combinación de ambos modelos presentó el mejor desempeño que las carteras optimizadas por solo uno de los modelos.

PALABRAS-CLAVE / Selección de portafolios, optimización multiobjetivo, Análisis de Grupo de Datos, indicadores, Índice Sharpe. 


\section{INTRODUÇÃO}

Considerando a estabilidade econômica observada no Brasil nos últimos anos e as consequentes quedas nos juros básicos da economia, acredita-se que opções de investimentos que busquem uma melhor rentabilidade no longo prazo devem atrair investidores nos próximos anos. Fundos de renda fixa e poupança, que representaram grande parte dos portfólios investidos nas últimas décadas, vêm perdendo atratividade, em função da queda das altas taxas de retornos presente um uma economia em estabilização, como é o caso da brasileira. Seguindo a tendência de mercados financeiros mais desenvolvidos, o investimento em ações vem se tornando uma boa alternativa de diversificação para investidores que buscam melhorar a rentabilidade de seu portfólio em longo prazo.

Os investimentos em ações tornam-se uma ótima alternativa quando comparados a outras aplicações, especialmente em prazos mais longos. Em geral, porém, essa maior rentabilidade é acompanhada de um determinado nível de risco. Dessa forma, objetivando a maximização do retorno, o investidor deve buscar as melhores formas de aplicar seu capital, evitando riscos maiores do que está disposto a aceitar.

As técnicas de composição de carteiras de ações vêm chamando a atenção não somente da academia como também do mercado financeiro, de acordo com Lopes, Lanzer e Lima (2006). Segundo Krokhmal, Zabarankin e Uryasev (2011), o trabalho de Markowitz (1952) estimulou o desenvolvimento de vários outros modelos de seleção de carteiras.

O modelo proposto por Markowitz (1952) é estático e indicado para avaliar um único período. Segundo Cover e Julian (2000) e Lopes, Lanzer e Lima (2006), encontram-se agora modelos dinâmicos ou de extensões multiperíodo. Belentepe (2005) acredita que o desempenho das técnicas mais desenvolvidas de seleção de carteiras parece não ter conseguido tão boa aceitação quanto as técnicas tradicionais, como a proposta de Markowitz (1952) e Sharpe $(1963,1964)$.

Nesse contexto, as técnicas de Pesquisa Operacional (PO), sobretudo a Análise Envoltória de Dados (Data Envelopment Analysis - DEA), têm demonstrado boa aplicação na área financeira, especialmente na avaliação de portfólios financeiros. É o que afirmam autores como Ceretta e Costa (2001), Haslem e Scheraga (2003), Gregoriou (2003) e Lopes, Lanzer e Lima (2006).

A DEA é uma técnica da PO desenvolvida por Charnes, Cooper e Rhodes (1978) e enriquecida por Banker, Charnes e Cooper (1984), considerada uma poderosa ferramenta gerencial, que, segundo Ceretta e Costa (2001) e Lopes, Lanzer e Lima (2006), é utilizada para avaliação e comparação de unidades organizacio- nais, destacando-se pelas vantagens operacionais oferecidas em avaliações multiatributos das unidades organizacionais.

Contextualizado o problema de pesquisa, o presente artigo tem como objetivo avaliar a eficiência de ações de empresas de capital aberto por meio da DEA.

Como objetivos específicos, tem-se:

- Montar uma carteira com todas as ações consideradas eficientes por meio da DEA;

- Utilizar a abordagem de Sharpe na montagem de uma carteira de ações consideradas eficientes por meio da DEA, a fim de determinar a alocação de capital que minimiza riscos para os investidores;

- Aplicar o modelo que apenas incorpora os conceitos da abordagem de Sharpe e compará-lo aos modelos que utilizam a técnica DEA.

\section{SELEÇÃO DE PORTFÓLIOS}

A teoria base da seleção de portfólios teve início com Markowitz (1952), em que a seleção de portfólio é baseada em um modelo de investimentos de período simples.

0 modelo proposto por Markowitz (1952), dado por (1) (3), é operacionalizado por técnicas de programação quadrática, cujo objetivo é otimização de carteiras levando-se em conta a média, a variância e a covariância dos retornos esperados das ações, que são opções para fazer parte da carteira. Esses parâmetros são estimados com base em informações de séries históricas, levando em conta um vetor de médias e uma matriz de covariância desses retornos.

$$
\min f(x)=\sum_{i=1}^{n} \sum_{j=1}^{n} \chi_{i} \chi_{j} \operatorname{cov}_{i j}
$$

Sujeito a:

$$
\sum_{i=1}^{j} \chi_{i} E_{(r i)}=E^{*}
$$

$$
\sum_{i=1}^{j} x_{i}=1
$$

No qual, $x_{i}$ e $x_{j}$ representam a participação percentual do ativo $\mathrm{i}$ e do ativo $j$ na carteira ótima, $\mathrm{E}_{(r i)}$ é o retorno esperado para o ativo de $i$, de $i=1$ a $j$, e $E^{\star}$ é o retorno esperado da carteira.

Segundo Abdelaziz, Aouni e Fayedh (2007), a metodologia de média-variância proposta por Markowitz (1952) para se- 
leção de portfólio tem sido fundamental para a atividade de pesquisa e tem servido como base para o desenvolvimento da teoria financeira moderna.

Na literatura, alguns algoritmos, como os propostos por Sharpe $(1963,1964)$ e Elton, Gruber e Padberg (1977), foram criados a fim de linearizar e melhorar a eficiência do modelo de covariância de Markowitz (Nawrocki \& Carter, 1998; Shing \& Nagasawa, 1999).

Os modelos clássicos são geralmente criticados como não eficientes. Com o objetivo de se criar um modelo mais eficiente, pesquisadores têm desenvolvido modelos mais sofisticados que utilizam extensões multiperiódicas ou dinâmicas (Cover \& Julian, 2000). Segundo Lopes, Carneiro e Schneider (2010), entretanto, passaram-se mais de 30 anos e as teorias de Markowitz (1952) e Sharpe $(1963,1964)$ continuam a embasar pesquisas sobre a gestão de portfólios.

\section{ANÁLISE ENVOLTÓRIA DE DADOS}

Segundo Lopes, Lanzer e Lima (2006), a técnica de medição de desempenho DEA, proposta por Charnes, Cooper e Rhodes (1978), consiste em determinar a eficiência relativa de uma unidade produtiva, considerando-se a aproximação de uma fronteira eficiente. Pode-se dizer que a DEA é um método não paramétrico de construção de uma fronteira eficiente, relativamente à qual se pode estimar a eficiência de cada unidade e determinar as unidades referenciais para os casos de ineficiência (Lopes, Lanzer \& Lima, 2006).

Segundo os autores, ainda, embora a maioria das aplicações DEA seja predominantemente sobre organizações sem fins lucrativos (Avkiran, 2001; Calhoun, 2003; Lopes, Lanzer, \& Lima 2006; Zhu, 2003), alguns trabalhos têm sido publicados na área de finanças, inclusive na otimização de portfólios (Chen, 2008; Emrouznejad, Parker, \& Tavares, 2008; Halim, 2010; Lopes, Lanzer, \& Lima 2006; Lopes, Carneiro \& Schneider, 2010).

O objetivo da DEA é determinar indicadores de desempenho relativo entre unidades produtivas, considerando certos conjuntos de insumos e produtos (inputs e outputs). Segundo Lopes, Lanzer e Lima (2006), esse é o único método de fácil utilização pelas empresas que possibilita avaliar a eficiência relativa de unidades que fabricam múltiplos produtos utilizando múltiplos insumos. De modo geral, pode-se dizer que os modelos DEA definem as melhores condições de operação para cada unidade produtiva separadamente, de modo a maximizar o seu índice de desempenho, e aplicam as mesmas condições às demais unidades da amostra em análise. Resolvendo-se o proble- ma para todas as unidades produtivas, obtêm-se as unidades produtivas que devem ser consideradas eficientes, que servirão de base para o estabelecimento da fronteira de eficiência e para o estabelecimento de metas as unidades ineficientes (Lopes, Lanzer, \& Lima, 2006).

Bal, Örkcü e Çelebioglu (2010) apontam que a DEA tem se destacado entre as modelagens quantitativas no auxílio à tomada de decisão pelos gestores. Charnes, Cooper e Rhodes (1978) abordaram esse tema pela primeira vez ao desenvolverem um modelo para uma nova medida de eficiência na avaliação de programas públicos. Comentam-se a seguir os modelos clássicos DEA: CCR e BCC.

Conforme Cooper, Seiford e Tone (2007), as variáveis de entrada e saída para cada Unidade Tomadora de Decisão - DMU (Decision Making Unit) devem atender alguns critérios, como:

As variáveis e DMUs devem ser escolhidas de modo a representar o interesse dos gestores;

Deve haver dados numéricos positivos para cada entrada e saída, devendo-se preferir um uso menor do número de entradas comparado ao de saídas.

Os pesos para variável de entrada e saída do modelo geral da DEA podem ser obtidos com base na solução do modelo proposto por Charnes, Cooper e Rhodes (1978), dado por (4) - (7):

$$
W_{0}=\max \frac{\sum_{r=1}^{s} u_{r} \cdot y_{r 0}}{\sum_{i=1}^{m} v_{i} \cdot X_{i 0}}
$$

Sujeito a:

$$
\frac{\sum_{r=1}^{s} u_{r} \cdot y_{r j}}{\sum_{i=1}^{m} v_{i} \cdot X_{i j}} \leq 1, j=1,2, \ldots, n
$$

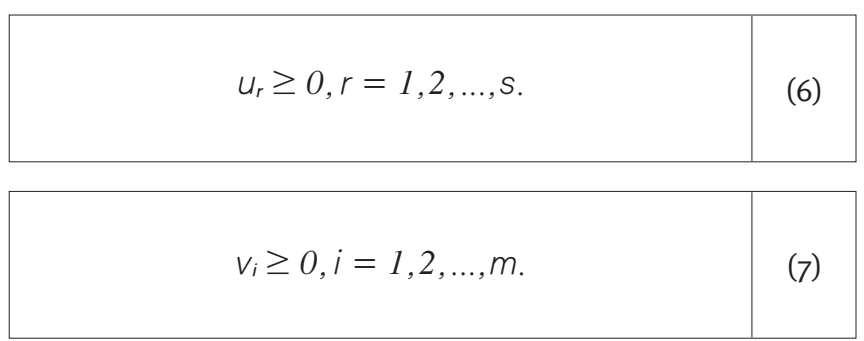

Com $j$ representando o índice da DMU, $j=1, \ldots, n$; $r$ é o índice da saída, com $r=1, \ldots, s$; $i$ é o índice da entrada, $i=1, \ldots, m$; $\mathrm{y}_{r j}$ é o valor da $r$-ésima saída para a j-ésima DMU; $\mathrm{x}_{i j}$ é o valor da $i$-ésima entrada para a j-ésima DMU; $u_{r}$ é o peso associado à $r$-ésima saída; $v_{i}$ é o peso associado à $i$-ésima entrada; $w_{o}$ é a eficiência relativa de $\mathrm{DMU}_{0}$, que é a DMU sob avaliação; e $\mathrm{y}_{r o} \mathrm{e}_{i o}$ 
são os coeficientes tecnológicos das matrizes de dados de saídas e entradas, respectivamente.

Caso $w_{o}=1$, a $D M U_{0}$ é eficiente quando comparada às demais unidades consideradas no modelo. Caso $w_{o}<1$, essa DMU é ineficiente. Esse modelo não é linear, sendo um caso da programação fracionária, mas que pode ser linearizado, conforme (8) - (12), pelo modelo conhecido por CCR, proposto por Charnes, Cooper e Rhodes (1978), ou com retornos constantes de escala:

$$
W_{0}=\max \sum_{r=1}^{s} u_{r} \cdot y_{r o}
$$

Sujeito a:

\begin{tabular}{c|c|}
$\sum_{i=1}^{m} v_{1} \cdot x_{i 0}=1$ & (9) \\
\hline$\sum_{r=1}^{s} u_{r} . y_{r 0}-\sum_{i=1}^{m} v_{i} . x_{i 0} \leq 0 j=1,2, \ldots, n$ & (10) \\
\hline$u_{r} \geq 0, r=1,2, \ldots, n$ & (11) \\
\hline$v_{i} \geq 0, i=1,2, \ldots, m$. & (12)
\end{tabular}

Sujeito a:

\begin{tabular}{|l|l|}
\hline$\sum_{i=1}^{m} v_{i} x_{i 0}=1$ & (14) \\
\hline
\end{tabular}

$$
\sum_{r=1}^{s} u_{r} y_{r 0}-\sum_{i=1}^{m} v_{i} x_{i 0}+c_{0} \leq 0 j=1,2, \ldots, n
$$

$$
u_{r} \geq 0, r=1,2, \ldots, s
$$

$$
v_{i} \geq 0, i=1,2, \ldots, m \text {. }
$$

A literatura recomenda, entretanto, que o número de DMUs seja igual a $1 / 3$ do número total de variáveis de entrada e saída. Quando tal cenário não ocorre, os modelos tradicionais de DEA (CCR e BCC) não proporcionam uma boa discriminação dos dados (Cooper, Seiford \& Tone, 2007).

\section{DESCRIÇÃO E MODELAGEM}

Segundo Bertrand e Fransoo (2002), esta pesquisa pode ser classificada como aplicada, tendo objetivo empírico descritivo, já que os pesquisadores estão interessados em criar um modelo que adequadamente descreve as relações causais que podem existir na realidade, conduzindo ao entendimento dos processos atuais, favorecendo, com isso, a compreensão de processos reais. A forma de abordar o problema foi quantitativa, sendo a modelagem matemática o método de pesquisa adotado.

Esta pesquisa utilizará a base científica desenvolvida por Markowitz para propor um método para seleção de portfólios de investimentos no mercado de ações. Para a seleção das ações eficientes, entretanto, será utilizado um modelo de DEA.

Aqui se descreve um método para seleção de portfólios de investimentos, aplicando o conceito proposto por Markowitz, com a incorporação de modelos matemáticos da PO comentados na seção anterior. A Figura 1 apresenta os passos que serão seguidos para condução desta pesquisa.

\section{Figura 1. Fluxograma da pesquisa}

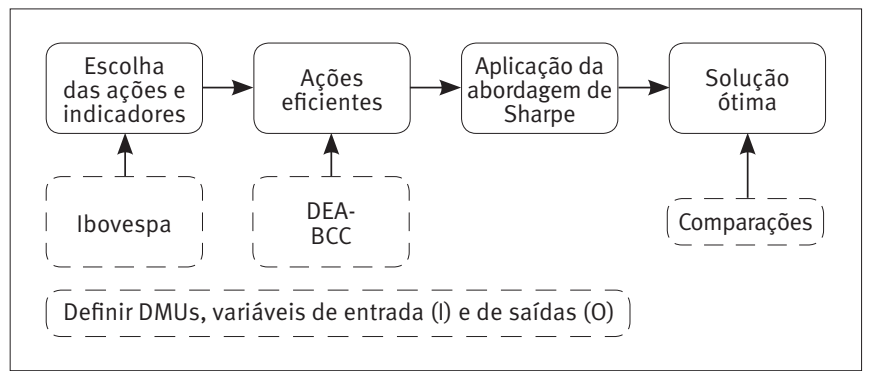

A amostra inicial foi constituída por ações de empresas de capital aberto na Bolsa de Valores de São Paulo (Bovespa), obtidas por meio de consultas ao banco de dados Economáti$\mathrm{ca}^{\circledR}$. Nesta pesquisa, optou-se pelas 40 empresas com maior participação no Ibovespa, sejam do tipo de ativos ON ou PN.

Após a definição da amostra, determinou-se o conjunto de indicadores de entrada e saída (inputs e outputs) utilizados na análise de eficiência por meio do modelo DEA.

Utilizaram-se os trabalhos de Powers e McMullen (2000), Lopes, Lanzer, e Lima (2006) e Lopes, Carneiro e Schneider (2010) como base para a definição de indicadores. Esses auto- 
res propõem que os indicadores de retorno de um, dois e três anos e lucro por ação compõem o conjunto de outputs, enquanto os indicadores como Beta (6o meses), preço/lucro e volatilidade (36 meses) compõem o conjunto de inputs. Segundo Powers e McMullen (2000), os indicadores buscam ativos que proporcionem os maiores valores de retornos e lucro por ação enquanto apresentam o menor preço/lucro e risco.

Analisando-se os trabalhos citados, verificou-se que os indicadores associados ao risco, Beta (6o meses) e volatilidade (36 meses), poderiam trazer informações imprecisas sobre o risco associado às carteiras comparativamente ao período abrangido pela rentabilidade. Visando fornecer ao modelo variáveis de entrada (inputs) que reflitam o risco associado aos períodos dos retornos analisados, propôs-se utilizar como indicadores de risco a volatilidade das ações selecionadas em prazos iguais aos utilizados nos retornos, em janelas de 12 meses, ou seja, anos 1, 2 e 3. Utilizando-se períodos iguais para risco e retorno, pretende-se, assim, fornecer ao modelo, variáveis de entrada (inputs) equivalentes quanto ao período das observações. Acredita-se que essa modificação no conjunto de indicadores da medida de risco pode trazer informações que permitirão ao modelo DEA selecionar ativos avaliados comparativamente como eficientes.

Ao decidir quais atributos devem ser classificados como outputs ou inputs, optou-se em favor do tratamento dos atributos com benefícios como saídas e o tratamento de atributos com custos como entradas.

Os dados foram coletados com o auxílio do software Economática ${ }^{\circledR}$. Dessa forma, este artigo visa avaliar a eficiência de ações (DMUs), em que as entradas ou inputs (I) são todos os indicadores que se deseja minimizar, e saídas ou outputs (0) são todos os indicadores que se deseja maximizar, como mostra a Tabela 1. Os dados negativos foram tratados conforme propõem Cook e Zhu (2008), sendo acrescidos do valor que tornava positivo o valor mais negativo, sem alterar a análise.

Neste trabalho, propõe-se um modelo DEA-BCC. Optou-se por tal modelo devido ao fato de as empresas atuarem em diferentes setores industriais, o que caracteriza um cenário de retorno variável de escala.

Para a modelagem do modelo DEA, utilizou-se o software The General Algebric Modeling (GAMS ${ }^{\circledR}$ ) na versão 23.6 .5 e solver CPLEX na versão 12.2.1.

Os resultados de eficiência (E) são apresentados pela última coluna da Tabela 1 a seguir. Os modelos considerados eficientes, ou seja, com valor igual a 1 no resultado, foram considerados participantes da carteira. Observa-se que, das 40 ações iniciais, apenas 15 foram consideradas eficientes de acordo com os indicadores selecionados.
Com o objetivo de comparação, três propostas de carteiras foram realizadas. Para isso, utilizaram-se os retornos mensais dos últimos 36 meses para cada um dos ativos, ou seja, de abril de 2010 a março de 2013. Os dados foram coletados com o software Economática ${ }^{\circledR}$ e posteriormente tratados, ou seja, nessa etapa, existiu a preocupação com descarte de outliers.

Na primeira delas, utilizou-se o suplemento Solver do Microsoft Excel ${ }^{\circledR}$ para otimizar os 40 ativos iniciais por meio da abordagem de Sharpe, obtendo os pesos recomendados para cada um dos 40 ativos, sendo denominada carteira Sharpe.

Para a segunda proposta, a mesma atividade foi realizada, entretanto foram considerados apenas os 15 ativos reconhecidos como eficientes pelo modelo DEA, ou seja, DMUs com valores de eficiência iguais a 1, sendo denominada carteira DEA Sharpe.

E, por fim, a terceira proposta, na qual se utilizaram os 15 ativos considerados eficientes por meio da DEA. O critério para alocação de capital foi, contudo, simplesmente a divisão por igual do montante a ser investido entre os ativos eficientes, como proposto por Lopes, Carneiro e Schneider (2010), sendo denominada carteira DEA $(1 / \mathrm{N})$.

Com as três propostas formuladas e otimizadas pelos critérios e restrições às quais foram submetidas, deu-se início ao processo de verificação da existência de retornos anormais. Para isso, utilizou-se a metodologia de verificação Capital Asset Pricing Model (CAPM) de Sharpe (1964), na qual foi possível comparar o portfólio no que diz respeito ao retorno esperado.

Para identificar retornos anormais dos ativos para cada uma das carteiras, calculou-se a diferença entre o retorno esperado fornecido pelo CAPM e o retorno observado no período de análise.

Segundo Assaf (2007), por meio da utilização do CAPM, além da obtenção da taxa de retorno, pode-se quantificar a relação entre risco e retorno à qual os ativos de uma empresa estão sujeitos. Com relação ao risco, o CAPM é constituído por dois pilares, o risco diversificável ou não sistemático e risco não diversificável ou sistemático.

Na equação do CAPM, para o retorno exigido para um ativo livre de risco (Rf), foi aplicada a taxa média da Selic referente ao período de abril de 2010 a março de 2013 , por ser considerado um indicador da taxa base de juros da economia com o risco do Tesouro Federal. Na verificação do desempenho do retorno esperado do mercado (Rm), observou-se o comportamento do Ibovespa a partir do início do ano de 1995 até o primeiro trimestre de 2013. O retorno esperado do mercado foi, então, calculado com base na média dos anos observados. 
TABELA 1. Dados de entradas (I) e saídas (0) e resultados da avaliação da eficiência

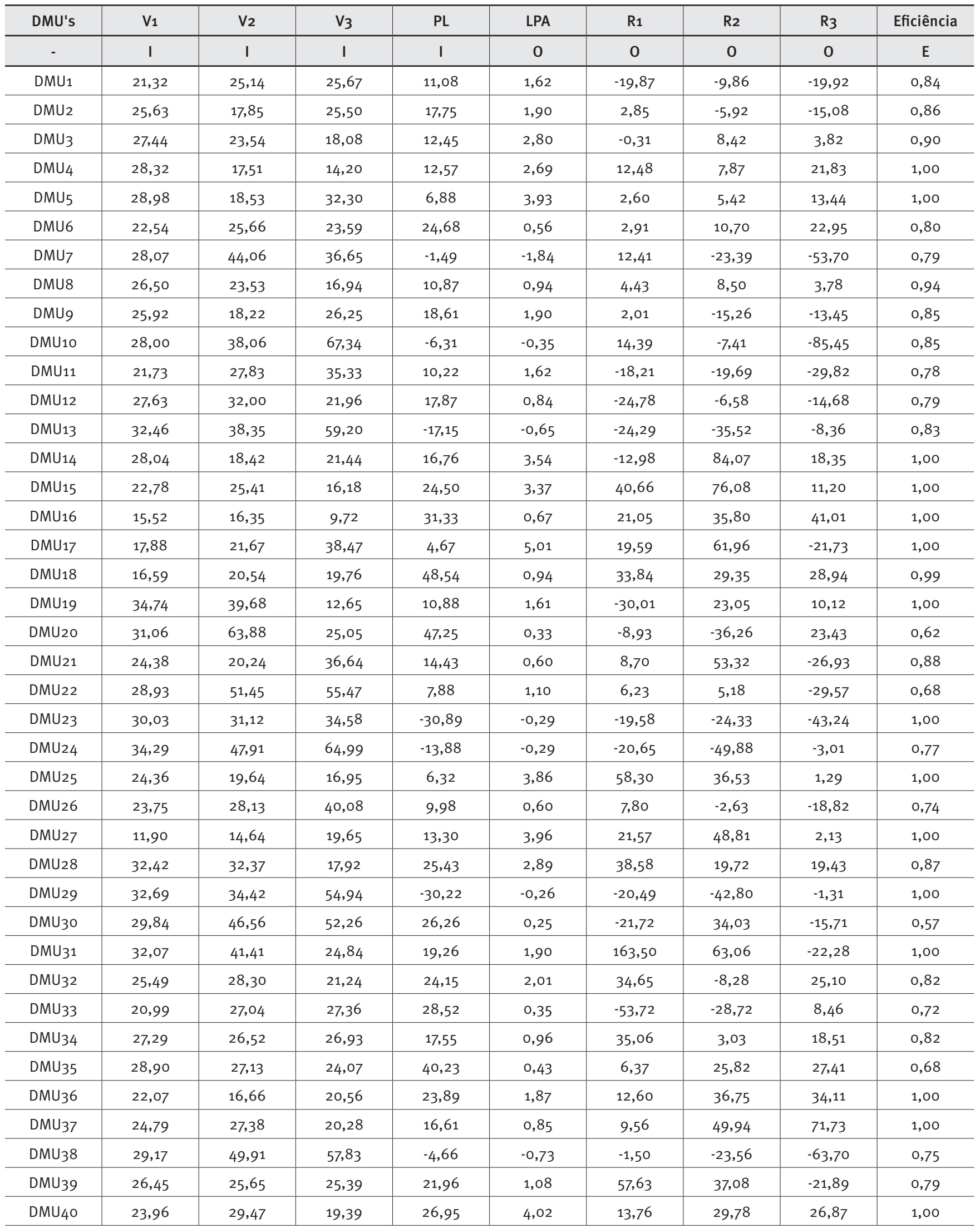


Utilizaram-se os valores de 9,6\% a.a. para taxa Selic média e $15,30 \%$ a.a. para (Rm) na definição da rentabilidade esperada das carteiras.

Por fim, calculou-se o termo Beta ( $\beta$ ), no qual, para cada uma das três carteiras formuladas, é calculado por meio da média ponderada do Beta individual multiplicado pelo percentual de cada papel. Para isso, obteve-se o Beta individual de cada um dos ativos, com auxílio do software Economática ${ }^{\circledR}$, durante o mesmo período utilizado para os retornos mensais. Foi possível, então, verificar se os portfólios resultaram em retornos acima dos esperados.

Para uma análise mais completa, também se utilizou o Índice de Sharpe (IS), que, segundo Paschoarelli (2008), é uma medida de eficiência no aproveitamento do risco para gerar retorno. 0 IS estabelece uma relação entre o excesso de rentabilidade de determinada carteira de investimento em relação à taxa de juros livre de risco e ao risco do investimento. Assumiuse como taxa de juros livre de risco a taxa média da Selic no período anteriormente proposto e, como risco do fundo, o desvio -padrão dos retornos mensais das carteiras.

\section{RESULTADOS E ANÁLISES}

0 resultado obtido foi a formação de três carteiras: uma utilizando a abordagem de Sharpe, que servirá como carteira comparativa (Sharpe), e outras duas que resultaram da seleção de ações eficientes utilizando a DEA. Com essa seleção de ações realizadas pelo DEA, utilizou-se a abordagem de Sharpe para criar uma carteira modelo (DEA Sharpe), na qual se buscou comparar, para os períodos selecionados, o comportamento da carteira com relação aos retornos obtidos e o risco medido pela variância, e uma terceira carteira, DEA $(1 / N)$, na qual se dividiu em iguais proporções o montante entre os ativos considerados eficientes por meio da DEA. Este artigo tem como objetivo avaliar o impacto do uso da metodologia DEA na pré-seleção de ações consideradas eficientes pelos indicadores propostos no estudo, submetendo esses mesmos ativos à abordagem de Sharpe, e verificar, por meio da comparação com as demais carteiras, a possível existência de benefícios ou diferenciais na utilização de tal proposta.

Na Tabela 2, são apresentados os resultados de variância, retorno e IS, obtidos com as carteiras propostas. Comparando-se os resultados obtidos pelo IS, apresentados na Tabela 2, pode-se observar que a carteira Sharpe apresentou IS positivo, de 0,280 , sendo composta por 13 ativos diferentes.

Já a carteira DEA $(1 / N)$ apresentou um IS de 0,187 , sendo composta pelos 15 ativos considerados eficientes por meio da DEA.

E, por fim, a carteira DEA Sharpe, na qual se avaliou a eficiência de 40 ações inicialmente propostas e obtiveram-se 15 ações consideradas eficientes. Otimizou-se, então, por meio da abordagem de Sharpe, obtendo a participação de cada um dos ativos a fim de maximizar o IS. A carteira otimizada é composta por apenas oito dos 15 ativos avaliados como eficientes e apresentou um IS de 0,584, demonstrando que o risco que oferece é recompensado, até mesmo superando os resultados das outras carteiras, mostrando-se um investimento atrativo.

Comparando-se os valores obtidos de IS em cada uma das carteiras, verificou-se a dominância da carteira DEA Sharpe sobre as demais, em que um investidor poderia obter um maior prêmio pelo risco assumido por esta.

Na Tabela 2, ainda são apresentados os valores de Beta $(\beta)$ de cada uma das três carteiras. Com os valores de Beta ( $\beta$ ), retorno esperado do mercado $(\mathrm{Rm})$ e retorno para um investimento livre de risco (Rf), foi possível calcular os valores de retorno esperado ( $R e$ ) das carteiras, também exibidos nesta tabela.

Pode-se observar que os retornos esperados das três carteiras apresentam valores semelhantes, em que a carteira Sharpe apresentou um (Re) de 0,92\% a.m., enquanto nas carteiras DEA $(1 / \mathrm{N})$ e DEA Sharpe obtiveram-se retornos esperados (Re) de 0,90\% a.m.

As rentabilidades efetivamente obtidas pelas carteiras Sharpe (1,49\% a.m.), DEA ( $1 / \mathrm{N})(1,54 \%$ a.m.) e DEA Sharpe $(2,43 \%$ a.m.) demonstram a existência de retornos anormais, quando comparadas as suas rentabilidades esperadas obtidas pelo modelo CAPM.

TABELA 2. Tabela de resultados

\begin{tabular}{c|c|c|c}
\hline & Sharpe & DEA (1/N) & DEA Sharpe \\
\hline Beta $(\beta)$ & 0,355 & 0,314 & 0,313 \\
\hline $\begin{array}{c}\text { Retorno } \\
\text { esperado (Re) }\end{array}$ & $0,92 \%$ & $0,90 \%$ & $0,90 \%$ \\
\hline Variância & $0,07 \%$ & $0,16 \%$ & $0,08 \%$ \\
\hline Retorno & $1,49 \%$ & $1,54 \%$ & $2,43 \%$ \\
\hline IS & 0,280 & 0,187 & 0,584 \\
\hline N. de ativos & 13 & 15 & 8 \\
\hline
\end{tabular}

A Tabela 3 mostra o retorno acumulado nos 36 meses em estudo obtido a partir da aplicação das três carteiras: Sharpe, DEA ( $1 / \mathrm{N})$ e DEA Sharpe. Comparam-se os retornos acumulados dessas carteiras com o do Ibovespa.

Por meio da Tabela 3 e da Figura 2, pode-se observar que as carteiras construídas superam o lbovespa. A carteira otimizada DEA Sharpe obtém o melhor desempenho, com um retorno acumulado de $127,29 \%$, superando as carteiras otimizadas apenas com a abordagem de Sharpe ou DEA. 
TABELA 3. Retorno acumulado dos modelos e do lbovespa

\begin{tabular}{|c|c|c|c|c|}
\hline Mês & Sharpe & $\operatorname{DEA}(1 / N)$ & $\begin{array}{c}\text { DEA } \\
\text { Sharpe }\end{array}$ & Ibovespa \\
\hline 1 & $0,00 \%$ & $0,00 \%$ & $0,00 \%$ & $0,00 \%$ \\
\hline 2 & $-2,89 \%$ & $-3,34 \%$ & $-2,31 \%$ & $-13,41 \%$ \\
\hline 3 & $-2,96 \%$ & $-1,95 \%$ & $0,03 \%$ & $-4,06 \%$ \\
\hline 4 & $2,05 \%$ & $8,84 \%$ & $6,00 \%$ & $-7,43 \%$ \\
\hline 5 & $2,91 \%$ & $8,88 \%$ & $9,51 \%$ & $-1,34 \%$ \\
\hline 6 & $8,38 \%$ & $14,88 \%$ & $15,36 \%$ & $0,43 \%$ \\
\hline 7 & $13,07 \%$ & $22,46 \%$ & $22,01 \%$ & $-3,79 \%$ \\
\hline 8 & $14,31 \%$ & $22,47 \%$ & $24,30 \%$ & $-1,52 \%$ \\
\hline 9 & $17,01 \%$ & $22,91 \%$ & $26,95 \%$ & $-5,40 \%$ \\
\hline 10 & $12,62 \%$ & $15,75 \%$ & $22,25 \%$ & $-4,25 \%$ \\
\hline 11 & $15,06 \%$ & $16,05 \%$ & $24,26 \%$ & $-2,53 \%$ \\
\hline 12 & $21,38 \%$ & $23,56 \%$ & $31,62 \%$ & $-6,02 \%$ \\
\hline 13 & $24,03 \%$ & $27,06 \%$ & $35,93 \%$ & $-8,17 \%$ \\
\hline 14 & $25,41 \%$ & $28,81 \%$ & $40,22 \%$ & $-11,32 \%$ \\
\hline 15 & $23,36 \%$ & $27,86 \%$ & $38,07 \%$ & $-16,41 \%$ \\
\hline 16 & $24,58 \%$ & $22,97 \%$ & $38,47 \%$ & $-19,72 \%$ \\
\hline 17 & $27,54 \%$ & $25,68 \%$ & $42,68 \%$ & $-25,65 \%$ \\
\hline 18 & $28,30 \%$ & $21,68 \%$ & $41,88 \%$ & $-17,10 \%$ \\
\hline 19 & $31,67 \%$ & $32,50 \%$ & $47,47 \%$ & $-19,18 \%$ \\
\hline 20 & $31,25 \%$ & $33,60 \%$ & $48,13 \%$ & $-19,35 \%$ \\
\hline 21 & $37,23 \%$ & $35,23 \%$ & $54,34 \%$ & $-10,38 \%$ \\
\hline 22 & $38,47 \%$ & $45,11 \%$ & $57,04 \%$ & $-6,49 \%$ \\
\hline 23 & $50,80 \%$ & $58,03 \%$ & $72,40 \%$ & $-8,34 \%$ \\
\hline 24 & $60,74 \%$ & $65,82 \%$ & $84,30 \%$ & $-12,16 \%$ \\
\hline 25 & $62,54 \%$ & $69,59 \%$ & $88,07 \%$ & $-22,58 \%$ \\
\hline 26 & $53,38 \%$ & $56,63 \%$ & $79,74 \%$ & $-22,77 \%$ \\
\hline 27 & $57,99 \%$ & $58,70 \%$ & $86,33 \%$ & $-20,29 \%$ \\
\hline 28 & $60,09 \%$ & $63,40 \%$ & $90,41 \%$ & $-18,92 \%$ \\
\hline 29 & $62,76 \%$ & $67,19 \%$ & $93,06 \%$ & $-15,92 \%$ \\
\hline 30 & $64,11 \%$ & $69,51 \%$ & $97,69 \%$ & $-18,92 \%$ \\
\hline 31 & $64,76 \%$ & $72,44 \%$ & $101,53 \%$ & $-18,34 \%$ \\
\hline 32 & $69,78 \%$ & $79,07 \%$ & $107,60 \%$ & $-13,40 \%$ \\
\hline 33 & $75,76 \%$ & $80,33 \%$ & $114,56 \%$ & $-15,09 \%$ \\
\hline 34 & $79,82 \%$ & $82,81 \%$ & $121,82 \%$ & $-18,41 \%$ \\
\hline 35 & $78,80 \%$ & $83,06 \%$ & $121,04 \%$ & $-19,94 \%$ \\
\hline 36 & $82,46 \%$ & $84,44 \%$ & $127,29 \%$ & $-21,78 \%$ \\
\hline
\end{tabular}

Observa-se que os resultados das carteiras DEA Sharpe e DEA $(1 / \mathrm{N})$ apresentam comportamento e retornos acumulados próximos, com $82,46 \%$ e $84,44 \%$ respectivamente. A carteira otimizada DEA $(1 / \mathrm{N})$, entretanto, apresenta uma pior relação no IS, quando comparada à carteira otimizada pela abordagem de Sharpe.
Figura 2. Retorno acumulado das carteiras otimizadas no período de 36 meses

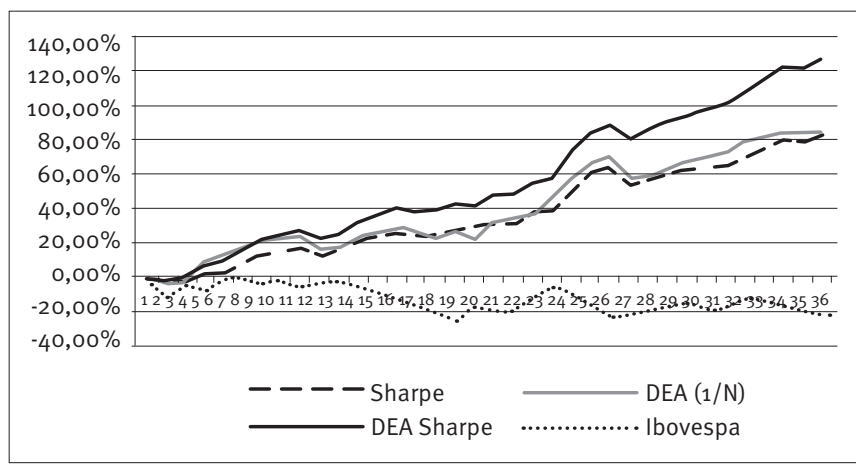

Nota: Baseada nos dados do Economática (2013)

\section{CONCLUSÕES}

Nesta pesquisa, avaliou-se a possibilidade da utilização da DEA na montagem de carteiras para investimentos na Bovespa. Para isso, três carteiras foram propostas: uma delas otimizada pela abordagem de Sharpe, uma segunda utilizando o DEA e considerando pesos iguais para os ativos considerados eficientes - DEA ( $1 / N)$, e outra na qual os pesos de ações consideradas eficientes foram otimizados pela abordagem de Sharpe - DEA Sharpe.

Buscou-se obter o modelo que apresenta melhor relação retorno/risco medida pelo IS, de modo a obter um melhor resultado do que a abordagem de Sharpe.

A aplicação da DEA mostrou-se viável e proporcionou uma excelente discriminação das unidades de análise. A carteira formada pela associação da DEA e da abordagem de Sharpe apresentou um melhor desempenho medido pelo IS, e, em termos de rentabilidade média mensal, superou a carteira comparativa em 29 dos 36 meses analisados.

Outro benefício observado é que, apesar do reduzido número de ações componentes da carteira DEA Sharpe (oito ações), o risco calculado da carteira ficou muito próximo ao risco calculado da carteira Sharpe (13 ações). Essa redução no número de ações, associada à manutenção do risco observado sob controle, tende a gerar economias referentes ao custo de rebalanceamento de carteiras, trazendo ganhos indiretos ao investidor.

A consagrada abordagem de Sharpe mostra-se eficiente em seu objetivo, entretanto, em algumas situações, obtém-se um grande número de ativos compondo a carteira e participações impossíveis de serem realizadas na prática, o que dificulta sua utilização.

A DEA já vem sendo considerada parte integrante de um conjunto de técnicas capazes de auxiliar na seleção de ativos 
para a composição de carteira. Já a combinação da DEA com a abordagem de Sharpe auxiliando na composição da carteira mostrou-se eficiente, e algumas vantagens podem ser citadas: utilizou-se um maior número de variáveis na análise, ou seja, uma melhor análise, que pode contemplar diferentes indicadores; como resultado, obteve-se um número reduzido de ativos compondo a carteira, o que torna viável sua utilização; e obteve-se o melhor IS entre as carteiras analisadas.

Como sugestão para futuras pesquisas, propõe-se a utilização de um maior número de indicadores e uma análise em longo prazo.

\section{NOTA DE AGRADECIMENTO}

Agradecemos à Fapemig, CNPq e CAPES pelo apoio financeiro e incentivo à pesquisa.

\section{REFERÊNCIAS}

Abdelaziz, F. ben, Aouni, B, \& Fayedh, R. el (2007). Multi-objective stochastic programming for portfolio selection. European Journal of Operational Research, 177(3), 1811-1823.

Assaf, A, Neto. (2007). Finanças corporativas e valor (3a ed.). São Paulo: Atlas.

Avkiran, N. K. (2001). Investigating technical and scale efficiencies of Australian universities through data envelopment analysis. Socio-Economic Planning Sciences, 35(1), 57-80.

Bal, H, Örkcü, H, \& Çelebioglu, S. (2010). Improving the discrimination power and weights dispersion in the data envelopment analysis. Computers \& Industrial Engineering, 37(1), 99-107.

Banker, R. D, Charnes, A, \& Cooper, W. W. (1984). Some models for estimating technical and scale inefficiencies in data envelopment analysis. Management Science, 30(9), 1078-1092.

Belentepe, C. (2005). A statistical view of universal portfolios. Doctoral dissertation, University of Pennsylvania.

Bertrand, J. W. M, \& Fransoo, J. C. (2002). Operations management research methodologies using quantitative modeling. International Journal of Operations \& Production Management, 22(2), 241-264.

Calhoun, J. (2003). Data envelopment analysis of relative efficiencies of institutions of higher learning. Portland: Association for the Study of Higher Education.

Ceretta, P, \& Costa, N, Jr. (2001). Avaliação e seleção de fundos de investimentos: um enfoque sobre múltiplos atributos. Revista de Administração Contemporânea, 5(1), 7-22.

Charnes, A, Cooper, W. W, \& Rhodes, E. (1978). Measuring the efficiency of decision-making units. European Journal of Operational Research, 2(6), 429-444.
Chen, H-H. (2008). Stock selection using data envelopment analysis. Industrial Management \& Data Systems, 108(9), 1255-1268.

Cook, W, \& Zhu, J. (2008). Data envelopment analysis: modeling operational processes and measuring productivity. Worcester: Create Space Independent Publishing Platform.

Cooper, W. W, Seiford, L. M, \& Tone, K. (2007). Data envelopment analysis: a comprehensive text with models, application, references and $D E A$-Solver Software (2. ed.). New York: Springer Science + Business.

Cover, T, \& Julian, D. (2000). Performance of universal portfolios in the stock market: information theory. Proceedings of IEEE International Symposium on Information Theory, Sorrento, 232.

Elton, E. J, Gruber, M. J, \& Padberg, M. (1977). Simple rules for optimal portfolio selection: the multi group case. Journal of Finance, 12(3), 329-345.

Emrouznejad, A, Parker, B, \& Tavares, G. (2008). Evaluation of research in efficiency and productivity: a survey and analysis of the first 30 years of scholarly literature in DEA. Social Economic Planning Sciences, 42(3), 151-157.

Gregoriou, G. (2003). The mortality of funds of hedge funds. Journal of Wealth Management, 5(4), 42-53.

Halim, R. E. (2010). Marketing productivity and profitability of Indonesian public listed manufacturing firms: an application of data envelopment analysis (DEA). Benchmarking: An International Journal, 17(6), 842-857.

Haslem, J, \& Scheraga, C. (2003). Data envelopment analysis of Morningstar's large-cap mutual funds. Journal of Investing, 12(4), 41-48.

Krokhmal, P, Zabarankin, M, \& Uryasev, S. (2011). Modeling and optimization of risk. Surveys in Operations Research and Management Science, 16(2), 49-66.

Lopes, A. L, Carneiro, M, \& Schneider, A. (2010). Markowitz na otimização de carteiras selecionadas por Data Envelopment Analysis - DEA. Revista Gestão e Sociedade, 4(9), 640-656.

Lopes, A. L, Lanzer, E, \& Lima, M. V. (2006). Avaliação do desempenho de carteiras de ações selecionadas pelo modelo de análise envoltória de dados - DEA. Anais do Congresso da Associação dos Analistas $e$ Profissionais de Investimento do Mercado de Capitais, Fortaleza, 19.

Markowitz, H. (1952). Portfolio selection. Journal of Finance, 7(1), 77-91.

Nawrocki, D. N, \& Carter, W. L. (1998). Earnings announcements and portfolio selection: do they add value? International Review of Financial Analysis, 7(1), 37-50.

Paschoarelli, R. (2008). Como ganhar dinheiro no mercado financeiro (2a ed.). São Paulo: Editora Elsevier.

Powers, J, \& McMullen, P. (2000). Using data envelopment analysis to select efficient large market cap securities. Journal of Business and Management, 7(2), 31-42.

Sharpe, W. F. (1963). A simplified model for portfolio analysis. Management Science, 9(2), 277-293.

Sharpe, W. F. (1964). Capital assets prices: a theory of market equilibrium under conditions of risk. Journal of Finance, 19(3), 425-442.

Shing, C, \& Nagasawa, H. (1999). Interactive decision system in stochastic multiobjective portfolio selection. International Journal of Production Economics, 60-61(20), 187-193.

Zhu, J. (2003). Quantitative models for performance evaluation and benchmarking. Massachusetts: Kluwer Academic Publishers. 